\title{
INTERDISCIPLINARIDADE ENTRE BIOLOGIA, GEOGRAFIA E ARTES: RELATO DE UMA EXPERIÊNCIA COM O ENSINO MÉDIO.
}

\author{
Mariana Spala Corrêa ${ }^{1}$ \\ Mariana Aparecida Lordeiro ${ }^{2}$ \\ Lorena Souza Castro ${ }^{3}$ \\ Esdras Santos ${ }^{4}$ \\ João Otávio da Silva Malaquias ${ }^{5}$ \\ Laís Amorim Ferreira ${ }^{6}$ \\ Erika Aparecida Silva de Freitas ${ }^{7}$ \\ Elias Terra Werner ${ }^{8}$
}

Resumo: A interdisciplinaridade como prática pedagógica contribui para a interação das disciplinas e contextualização dos conteúdos. Entretanto, a maioria dos docentes encontram dificuldades para realizar essas atividades. O presente trabalho teve por objetivo analisar o desempenho de duas turmas da $1^{a}$ série do ensino médio, sobre o ciclo da água, com o intuito de identificar se a interdisciplinaridade adotada como método cotidiano de ensino motiva os discentes, desperta seu interesse e contribui para uma melhor aprendizagem. Por meio de ações interdisciplinares que integram as disciplinares de Biologia, Geografia e Artes, oferecemos aos alunos da turma A uma aula demonstrativa, com a utilização do flanelógrafo e contextualização. E a aplicação de uma aula expositiva para a turma $B$, sem a utilização de outros instrumentos didáticos e metodologia interdisciplinar. Observamos que a turma $A$ apresentou melhor rendimento, o que contribuiu para maior socialização e participação dos alunos.

Palavras-chave: Interdisciplinaridade; Prática pedagógica; Ensino médio; Contextualização.

\footnotetext{
${ }^{1}$ Graduanda em Ciências Biológicas Licenciatura/Departamento de Biologia/Centro de Ciências Exatas, Naturais e da Saúde/Universidade Federal do Espirito Santo - UFES, Brasil. E-mail: marianaspala@gmail.com.

${ }^{2}$ Graduanda em Ciências Biológicas Licenciatura/Departamento de Biologia/Centro de Ciências Exatas, Naturais e da Saúde/Universidade Federal do Espirito Santo - UFES, Brasil. E-mail: mariana.lordeiro@gmail.com.

${ }^{3}$ Graduanda em Ciências Biológicas Licenciatura/Departamento de Biologia/Centro de Ciências Exatas, Naturais e da Saúde/Universidade Federal do Espirito Santo - UFES, Brasil. E-mail: Iorena-sc3@hotmail.com.

${ }^{4}$ Graduando em Ciências Biológicas Licenciatura/Departamento de Biologia/Centro de Ciências Exatas, Naturais e da Saúde/Universidade Federal do Espirito Santo - UFES, Brasil. E-mail: Iorena-sc3@hotmail.com; esdrasgutemberg@hotmail.com.

${ }^{5}$ Graduando em Ciências Biológicas Licenciatura/Departamento de Biologia/Centro de Ciências Exatas, Naturais e da Saúde/Universidade Federal do Espirito Santo - UFES, Brasil. E-mail: joaootaviosm@gmail.com.

${ }^{6}$ Graduanda em Ciências Biológicas Licenciatura/Departamento de Biologia/Centro de Ciências Exatas, Naturais e da Saúde/Universidade Federal do Espirito Santo - UFES, Brasil. E-mail: joaootaviosm@gmail.com. laisguvi@hotmail.com.

Docente/Escola Estadual de Ensino Fundamental e Médio Aristeu Aguiar, Brasil. E-mail: erikasfbr@yahoo.com.br.

${ }^{8}$ Professor Doutor/Departamento de Biologia/Centro de Ciências Exatas, Naturais e da Saúde/Universidade Federal do Espirito Santo - UFES, Brasil. E-mail: eliaswerner12@gmail.com.
} 